\title{
Efeito da radiação vermelha de baixa intensidade sobre o sêmen canino criopreservado
}

\author{
[Effect of low intensity red light on the canine semen cryopreservation] \\ M.F. Brito ${ }^{1}$, T.T.C. Carvalho $^{1}$, G.R. Valle ${ }^{1}$, L.F.L. Ferreira ${ }^{1}$, J.V.M. Mambrini ${ }^{2}$, \\ M.R.J.M. Henry ${ }^{3}$, M.I.V. Melo ${ }^{1 *}$ \\ ${ }^{1}$ Pontifícia Universidade Católica de Minas Gerais - Betim, MG \\ ${ }^{2}$ Consultor Estatístico - Belo Horizonte, MG \\ ${ }^{3}$ Escola de Veterinária - Universidade Federal de Minas Gerais - Belo Horizonte, MG
}

\begin{abstract}
RESUMO
O objetivo geral deste trabalho foi analisar o efeito da radiação vermelha de baixa intensidade sobre alguns parâmetros cinéticos do espermatozoide canino criopreservado. Ejaculados de oito cães adultos foram centrifugados, rediluídos em meio tris-gema de ovo com 6\% de glicerol, e, posteriormente, fracionados em: T1: incidência de radiação vermelha (660 NM) (Fisioled - Mmoptics - 100mW) por 60 segundos, antes do resfriamento e após a descongelação; T2: incidência somente antes do resfriamento; T3: incidência somente após a descongelação; e T4: sem incidência. Após a descongelação, as amostras foram submetidas ao TTR utilizando-se Sperm Class Analyzer®. No TTR0, TTR60 e TTR90, não houve diferença entre as variáveis analisadas pelo CASA. Somente no TTR30 os efeitos da incidência da radiação vermelha foram evidentes e significativos em T1 e T2; T1 resultou em baixa MT $(12,5+10,6 \%)$ e T2 determinou o melhor resultado de MT 40,3 + 26,1\%. De forma similar T1 apresentou maior número de espermatozoides estáticos $(77,5 \pm 28,9 \%)$ em relação ao T2 $(50,6 \pm 28 \%)$. Concluiu-se que a dupla incidência de radiação vermelha de baixa intensidade antes do resfriamento e após a descongelação teve efeito deletério sobre a motilidade do espermatozoide canino, expressa principalmente aos 30 minutos após descongelação.
\end{abstract}

Palavras-chave: cão, criopreservação, radiação vermelha, CASA

\begin{abstract}
The aim of this study was to analyze the effect of low intensity red light on some kinetic parameters of cryopreserved canine sperm. Ejaculates from 08 adult dogs were centrifuged, diluted in Tris-egg yolk with 6\% glycerol, and subsequently separated into: T1: incidence of red light (660 nm) (Fisioled MMOptics - 100mW) for 60 seconds before the cooling and after thawing, T2: just before cooling, T3: only after thawing, and T4: no incidence. After thawing the samples were subjected to TTR using Sperm analyzer ${ }^{\circledR}$. In TTRO, TTR60 and TTR90 there were no differences between the variables analyzed by CASA. Only in TTR30 the effects of the incidence of red light were visible and significant in T1 and T2. $T 1$ resulted in low MT $(12.5 \pm 10.6 \%)$ and T2 determined the best result of MT 26.1\% \pm 40.3 . Similarly T1 showed a higher number of static spermatozoa $(77.5 \pm 28.9 \%)$ compared to T2 $(50.6 \pm 28 \%)$. We concluded that the double incidence of low intensity red light, before cooling and after thawing, had a deleterious effect on canine sperm motility expressed at 30 minutes after thawing.
\end{abstract}

Keywords: dog, cryopreservation, red radiation, CASA

Recebido em 3 de março de 2013

Aceito em 25 de junho de 2014

*Autor para correspondência (corresponding author)

E-mail: bel.melo@terra.com.br 


\section{INTRODUÇÃO}

As biotecnologias reprodutivas, como o congelamento de sêmen, permitiram o avanço genético de raças, por meio da troca de material entre localidades distantes, bem como a preservação de germoplasma de espécies em processo de extinção, ou de interesse zootécnico.

Para o sucesso da técnica de inseminação artificial, um fator determinante é a qualidade seminal pós-descongelamento. Dessa forma, são necessários alguns cuidados, como: adição de um bom diluidor, binômios de tempo e temperatura de congelação-descongelação corretos, armazenamento e manipulação adequados. Além disso, os últimos estudos têm apontado para a possibilidade de melhorar os parâmetros espermáticos e, assim, a qualidade do sêmen, por meio da radiação vermelha de baixa intensidade (Ocaña-Quero et al., 1997; Cohen et al., 1998; Corral-Baqués et al., 2005; Laffaldano et al., 2005; Laffaldano et al., 2007; Brandão, 2008).

O uso da radiação sobre células espermáticas foi proposto com o intuito de aumentar a motilidade de espermatozoides e, consequentemente, sua capacidade de fertilização. Acredita-se que a radiação interfira na cadeia respiratória das mitocôndrias, aumentando a produção de energia (ATP), bem como no conteúdo celular de aminoácidos livres, causando incremento de motilidade, induzindo reação acrossomal e reduzindo a mortalidade de células espermáticas in vitro (Ocaña-Quero et al., 1997; Laffaldano et al., 2007; Corral-Baqués et al., 2009).

Trabalhos encontrados na literatura se referem à utilização do laser (Light Amplification Stimulation Emission Radiation) como fonte de radiação de baixa intensidade em diferentes espécies, com o objetivo de melhoria da qualidade seminal. Porém, nesses estudos, não se observou homogeneidade dos resultados, sendo estes, por vezes, controversos. Estudos com a utilização do LED (Light Emission Diode), como fonte de radiação de baixa intensidade, não foram encontrados na literatura pesquisada. Por esse ser um aparelho recentemente empregado na medicina veterinária, de mais baixo custo e menor consumo de energia, além de estar sendo empregado em outras terapias, com êxito, em substituição ao uso do laser, propôs-se estudar o efeito do LED sobre a qualidade do sêmen de cães, aplicando-o em diferentes momentos.

\section{MATERIAL E MÉTODOS}

O trabalho foi desenvolvido entre os meses de fevereiro de 2010 e maio de 2011. O sêmen foi obtido de oito cães adultos de diferentes raças (Bull Mastif, Pastor Canadense, Scothish Terrier, SRD, Buldogue Inglês e Golden Retriever), mantidos sob manejo padronizado, de canil particular/comercial da cidade de Betim, com idades variando de um a três anos.

O método de coleta realizado foi a manipulação peniana com auxílio de luva de palpação, cortada e vedada (com uso de seladora) de modo a formar um "cone", ao final do qual foi acoplado um tubo falcon acoplado (Valle et al., 2012). Por essa metodologia, as frações de sêmen foram coletadas até que se observasse a ejaculação da terceira fração, sendo o ejaculado imediatamente submetido à centrifugação. Coletou-se um ejaculado de cada cão, e cada ejaculado foi considerado uma repetição, totalizando oito repetições. Cada ejaculado foi centrifugado a $800 \mathrm{G} / 15$ minutos. Removeu-se o sobrenadante e realizou-se rediluição em meio Tris-Gema de ovo, aquecido a $37^{\circ} \mathrm{C}$, com $6 \%$ de glicerol, visando ao volume final de $6 \mathrm{~mL}$.

O referido experimento, com a descrição de todos os procedimentos que seriam realizados com os animais, foi submetido ao Comitê de Ética no uso de Animais da PUC Minas (CEUA PUC Minas - Protocolo 019) e aprovado.

A fonte de radiação vermelha utilizada foi o LED de baixa intensidade (diodo emissor de luz), com $660 \mathrm{~nm}$ de comprimento de onda (Fisioled Mmoptics) com potência de $100 \mathrm{~mW}$. Foi estabelecida densidade de energia (dose) de 6 $\mathrm{J} / \mathrm{cm}^{2}$ durante $60 \mathrm{seg}$, sendo realizadas duas incidências, em sequência, de mesma dose e tempo. Todas as irradiações foram feitas nas amostras em um becker de vidro de $50 \mathrm{~mL}$ (de $5 \mathrm{~cm}$ de diâmetro) a uma distância, entre ponteira do aparelho e coluna de líquido (sêmen), de $4,5 \mathrm{~cm}$. O becker foi envolto com um emborrachado preto a fim de evitar a exposição do manipulador e a perda de energia através do vidro. 
Os tratamentos estabelecidos foram: incidência de radiação vermelha antes do resfriamento e após a descongelação (T1); incidência de radiação vermelha apenas antes do resfriamento (T2); incidência de radiação apenas após a descongelação (T3); sem incidência de radiação vermelha (GC).

Os tubos falcon foram colocados em becker de $50 \mathrm{~mL}$ contendo $30 \mathrm{~mL}$ de água à temperatura ambiente, a fim de retardar a queda de temperatura, proporcionando uma curva de resfriamento de $0,14^{\circ} \mathrm{C} / \mathrm{min}$. Após duas horas de curva, os tubos foram mantidos no vapor da caixa $\left(5^{\circ} \mathrm{C}\right)$ para tempo de equilíbrio de $60 \mathrm{~min}$. Após esse tempo, as amostras foram envasadas em palhetas de $0,25 \mathrm{~mL}$ e congeladas segundo metodologia descrita por Silva et al. (2003). As palhetas foram dispostas horizontalmente em uma caixa térmica de isopor contendo nitrogênio líquido por 10 minutos, a $5 \mathrm{~cm}$ acima do nível de nitrogênio, e, então, mergulhadas no nitrogênio e armazenadas à temperatura de $-196^{\circ} \mathrm{C}$, mantidas por um período mínimo de sete dias antes de serem descongeladas. Todas as palhetas foram descongeladas a $38^{\circ} \mathrm{C}$ por 30 segundos.

As amostras foram avaliadas subjetivamente quanto à motilidade espermática total (MT), à motilidade espermática progressiva (MP) e ao vigor espermático (V) imediatamente após a coleta do sêmen e após a incidência da radiação vermelha, antes do resfriamento.

Após a descongelação, as amostras também foram avaliadas quanto à longevidade espermática usando-se o teste de termorresistência (TTR) (Manual..., 1998), com leitura feita em sistema computadorizado de análise de sêmen nos tempos de 0min, 30min, $60 \mathrm{~min}$ e 90min pós-descongelação. O equipamento utilizado foi o Sperm Class Analyser ${ }^{\circledR}(\mathrm{SCA} \circledast)$, acoplado a um microscópio de contraste de fase. No mínimo três imagens de cada amostra foram obtidas para análise. Os parâmetros de motilidade estudados foram: velocidade curvilinear (VCL), velocidade progressiva (VSL), velocidade média da trajetória (VAP), retilinearidade (STR), coeficiente linear (LIN), deslocamento lateral da cabeça (ALH), frequência de batimento flagelar cruzado (BCF), espermatozoides estáticos, com motilidade progressiva, não progressiva e total, espermatozoides de velocidade rápida, média e lenta.

A análise estatística realizada foi primeiramente descritiva, apresentando os valores de média e desvio-padrão da motilidade total, progressiva e do vigor das leituras subjetivas realizadas imediatamente após a coleta e após a radiação antes do resfriamento.

De forma similar, todos os parâmetros de motilidade analisados pelo CASA, durante o período de zero a 90mim pós-descongelação, foram descritivamente apresentados (média, desvio-padrão), em intervalos de 30 minutos. O delineamento experimental foi de blocos ao acaso, considerando o cão como bloco. Entretanto, os valores obtidos não apresentaram distribuição normal, e, por isso, empregou-se análise não paramétrica.As médias foram comparadas pelo teste de Kruskal-Wallis em cada tempo (0min, 30min, 60min e 90min pósdescongelação).

Quando se observou diferença significativa entre variáveis, os valores médios foram comparados de 2 a 2 pelo teste de Mann-Whitney. Para o nível de significância, foi feito o índice de correção de Bonferroni, alterando o nível de significância (p) a fim de evitar erros derivados da múltipla comparação.

\section{RESULTADOS E DISCUSSÃO}

As médias dos valores de MT, MP e V, analisadas subjetivamente após a coleta de sêmen, foram, respectivamente, $76,9 \pm 12,5 \%$,

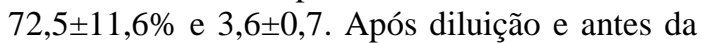
incidência de radiação, os valores médios foram $73,1 \pm 10,7 \%, 66,9 \pm 11,3 \%$ e $3 \pm 0,6$. Esses valores estão próximos dos valores descritos para a espécie canina em relação a sêmen fresco (Bueno, 2000).

Imediatamente após o descongelação (tempo 0 min), não houve diferença significativa entre os diferentes tratamentos $(\mathrm{P}>0,05)$, em nenhum dos parâmetros analisados pelo CASA (Tab. 1). Em estudo da incidência de radiação laser em sêmen de peru, Laffaldano et al. (2005) também não encontraram diferenças entre o grupo irradiado e o controle nas amostras avaliadas imediatamente após a incidência. Os autores atribuíram essa falta de efeito positivo ao fato de a 
bioestimulação requerer um período de tempo mínimo para que a célula possa obter do meio metabólitos essenciais, que são necessários às reações em que a irradiação está envolvida, percebido na leitura de motilidade. Entretanto, Corral-Baqués et al. (2009), ao analisarem sêmen fresco de cães irradiados com diferentes potências de laser He-Ne, numa dose de $3,34 \mathrm{~J} / \mathrm{cm}^{2}$, encontraram aumentos significativos nos valores de VCL, VSL, VAP, LIN e WOB, imediatamente após a incidência, sendo esta questão do efeito, ser imediato ou não, controversa.

Tabela 1. Médias ( \pm DP) dos parâmetros analisados pelo CASA, para espermatozoides caninos criopreservados, processados com e sem irradiação LED, imediatamente após a descongelação (tempo 0 $\min )$

\begin{tabular}{|c|c|c|c|c|c|c|c|c|}
\hline Características & \multicolumn{2}{|r|}{$\mathrm{T} 1$} & \multicolumn{2}{|r|}{$\mathrm{T} 2$} & \multicolumn{2}{|r|}{ T3 } & \multicolumn{2}{|r|}{$\mathrm{GC}$} \\
\hline Static & 61,92 & $\pm 25,12$ & 43,4 & $\pm 28,19$ & 55,63 & $\pm 24,63$ & 41,38 & $\pm 33,18$ \\
\hline NP & 29,8 & $\pm 16,62$ & 45,78 & $\pm 21,83$ & 35,13 & $\pm 17,31$ & 44,15 & $\pm 22,80$ \\
\hline $\mathrm{P}$ & 8,28 & $\pm 10,49$ & 10,82 & $\pm 11,36$ & 9,24 & $\pm 9,75$ & 14,48 & $\pm 11,91$ \\
\hline MT & 38,08 & $\pm 25,12$ & 56,6 & $\pm 28,19$ & 44,37 & $\pm 24,63$ & 58,62 & $\pm 33,18$ \\
\hline Rapid & 3,05 & $\pm 5,25$ & 5,12 & $\pm 7,16$ & 2,68 & $\pm 3,36$ & 7,87 & $\pm 10,40$ \\
\hline Med & 6,31 & $\pm 7,44$ & 8,7 & $\pm 7,84$ & 7,96 & $\pm 8,27$ & 11,04 & $\pm 10,80$ \\
\hline Slow & 28,72 & $\pm 15,45$ & 42,78 & $\pm 18,82$ & 33,73 & $\pm 15,96$ & 39,72 & $\pm 18,99$ \\
\hline VCL & 34,7 & $\pm 11,92$ & 37,57 & $\pm 12,83$ & 38,03 & $\pm 9,52$ & 42,63 & $\pm 16,19$ \\
\hline VSL & 20,79 & $\pm 8,66$ & 20,82 & $\pm 9,39$ & 23,5 & $\pm 7,85$ & 24,6 & $\pm 9,50$ \\
\hline VAP & 25 & $\pm 9,93$ & 26,15 & $\pm 10,18$ & 28,09 & $\pm 8,46$ & 30,2 & $\pm 11,78$ \\
\hline LIN & 59,22 & $\pm 7,20$ & 54,96 & $\pm 12,55$ & 61,15 & $\pm 13,76$ & 58,15 & $\pm 7,86$ \\
\hline STR & 82,82 & $\pm 3,21$ & 78,52 & $\pm 8,83$ & 82,62 & $\pm 7,09$ & 81,73 & $\pm 5,74$ \\
\hline ALH & 2,11 & $\pm 1,28$ & 2,47 & $\pm 1,11$ & 1,96 & $\pm 1,12$ & 2,6 & $\pm 1,20$ \\
\hline $\mathrm{BCF}$ & 9,09 & $\pm 5,26$ & 9,75 & $\pm 3,97$ & 8,98 & $\pm 5,08$ & 9,89 & $\pm 4,24$ \\
\hline
\end{tabular}

T1: incidência de radiação vermelha antes do resfriamento e após a descongelação; T2: incidência de radiação vermelha apenas antes do resfriamento; T3: incidência de radiação apenas após a descongelação; GC: sem incidência de radiação vermelha. Static - espermatozoides estáticos; NP - motilidade não progressiva; $\mathrm{P}$ - motilidade progressiva; MT - motilidade total; Rapid - espermatozoides rápidos; Med - espermatozoides com velocidade média; slow - espermatozoides com velocidade lenta; VCL - velocidade curvilinear; VSL - velocidade progressiva; VAP - velocidade média do trajeto; LIN - linearidade; STR - retilinearidade; ALH - amplitude do deslocamento lateral da cabeça; $\mathrm{BCF}$ - frequência de batimento flagelar. $(\mathrm{P}>0,05)$

(Fonte: SCA® - Avaliação gerada pelos autores).

$\mathrm{Na}$ Tab. 2, podem ser observadas diferenças entre os tratamentos com incidência de radiação antes do resfriamento e após a descongelação (T1) e o tratamento com incidência apenas antes do resfriamento (T2), na avaliação aos 30 minutos após a descongelação. Essas diferenças foram significativas nos parâmetros de MT, número de espermatozoides estáticos, motilidade não progressiva e espermatozoides lentos $(\mathrm{p}=$ 0,004). Esses resultados sugerem que a incidência de radiação antes do resfriamento e após a descongelação provoca efeitos deletérios na motilidade do espermatozoide canino em relação à incidência apenas antes do resfriamento. Acredita-se que esse achado seja devido a um processo de aceleração do envelhecimento da mitocôndria, por uma superestimulação da respiração mitocondrial, uma vez que a radiação luminosa provoca um aumento na produção de ATP (Laffaldano et al., 2005). Dessa forma, quando a célula espermática foi submetida a duas séries de radiação (mesmo que em momentos diferentes), foi observado significativo declínio da MT espermática ao longo do tempo (leitura aos 30 minutos após a incidência de radiação), reduzindo a velocidade de deslocamento e, inclusive, determinando maior percentual de morte celular, representado por um maior número de espermatozoides estáticos. O número de espermatozoides estáticos do grupo tratado com radiação antes do resfriamento e após a descongelação (T1) também foi significativamente maior que aqueles tratados sem a incidência de radiação (T4), reforçando a ideia de aceleração do envelhecimento mitocondrial. 


\section{Brito et al.}

Tabela 2. Médias ( \pm DP) dos parâmetros analisados pelo CASA, para espermatozoides caninos criopreservados, processados com e sem irradiação LED, 30 minutos após a descongelação

\begin{tabular}{|c|c|c|c|c|c|c|c|c|}
\hline Características & & $\mathrm{T} 1$ & & $\mathrm{~T} 2$ & & T3 & & GC \\
\hline Static & 77,53 & $\pm 28,91 \mathrm{a}$ & 50,63 & $\pm 27,99 \mathrm{~b}$ & 66,37 & $\pm 30,33 \mathrm{ab}$ & 45,05 & $\pm 35,11 b$ \\
\hline NP & 10,47 & $\pm 7,58 \mathrm{a}$ & 34,49 & $\pm 20,92 b$ & 21,27 & $\pm 17,75 \mathrm{ab}$ & 31,44 & $\pm 27,05 \mathrm{ab}$ \\
\hline $\mathrm{P}$ & 2 & $\pm 3,57$ & 5,79 & $\pm 7,17$ & 3,27 & $\pm 5,23$ & 5,33 & $\pm 6,49$ \\
\hline MT & 12,47 & $\pm 10,63 \mathrm{a}$ & 40,28 & $\pm 26,07 b$ & 24,54 & $\pm 22,40 \mathrm{ab}$ & 36,77 & $\pm 32,67 \mathrm{ab}$ \\
\hline Rapid & 0,37 & $\pm 0,93$ & 1,15 & $\pm 1,89$ & 1,59 & $\pm 3,55$ & 2,13 & $\pm 3,47$ \\
\hline Med & 1,76 & $\pm 2,93$ & 5,58 & $\pm 7,00$ & 2,52 & $\pm 3,73$ & 5,49 & $\pm 7,68$ \\
\hline Slow & 10,33 & $\pm 7,45 \mathrm{a}$ & 33,54 & $\pm 19,85 b$ & 20,43 & $\pm 16,19 \mathrm{ab}$ & 29,16 & $\pm 23,49 \mathrm{ab}$ \\
\hline VCL & 22,65 & $\pm 13,33$ & 26,04 & $\pm 14,22$ & 26,49 & $\pm 13,09$ & 26,27 & $\pm 16,44$ \\
\hline VSL & 11,93 & $\pm 9,38$ & 12,57 & $\pm 9,33$ & 12,91 & $\pm 7,46$ & 11,63 & $\pm 7,96$ \\
\hline VAP & 14,9 & $\pm 10,64$ & 16,38 & $\pm 10,97$ & 16,89 & $\pm 9,09$ & 15,95 & $\pm 10,80$ \\
\hline LIN & 45,18 & $\pm 23,28$ & 41,02 & $\pm 22,52$ & 43,29 & $\pm 18,91$ & 35,43 & $\pm 19,90$ \\
\hline STR & 68,46 & $\pm 27,22$ & 65,4 & $\pm 25,29$ & 67,79 & $\pm 24,62$ & 59,26 & $\pm 30,09$ \\
\hline ALH & 1,54 & $\pm 1,38$ & 2,56 & $\pm 1,19$ & 1,79 & $\pm 1,66$ & 2,56 & $\pm 1,41$ \\
\hline $\mathrm{BCF}$ & 4,84 & $\pm 4,47$ & 7.34 & \pm 4.00 & 5,36 & $\pm 5,15$ & 8,26 & $\pm 4,28$ \\
\hline
\end{tabular}

T1: incidência de radiação vermelha antes do resfriamento e após a descongelação; T2: incidência de radiação vermelha apenas antes do resfriamento; T3: incidência de radiação apenas após a descongelação; GC: sem incidência de radiação vermelha. Static - espermatozoides estáticos; NP - motilidade não progressiva; P - motilidade progressiva; MT - motilidade total; Rapid - espermatozoides rápidos; Med - espermatozoides com velocidade média; Slow - espermatozoides com velocidade lenta; VCL - velocidade curvilinear; VSL - velocidade progressiva; VAP - velocidade média do trajeto; LIN - linearidade; STR - retilinearidade; ALH - amplitude do deslocamento lateral da cabeça; $\mathrm{BCF}$ - frequência de batimento flagelar.

$\mathrm{a}, \mathrm{b}$ - diferentes letras indicam diferença significativa entre os tratamentos $(\mathrm{P}<0,004)$.

(Fonte: SCA® - Avaliação gerada pelos autores).

Tabela 3. Médias ( \pm DP) dos parâmetros analisados pelo CASA, para espermatozoides caninos criopreservados, processados com e sem irradiação LED, 60 minutos após a descongelação

\begin{tabular}{|c|c|c|c|c|c|c|c|c|}
\hline Características & \multicolumn{2}{|r|}{ T1 } & \multicolumn{2}{|r|}{$\mathrm{T} 2$} & \multicolumn{2}{|r|}{ T3 } & \multicolumn{2}{|c|}{ GC } \\
\hline Static & 39,31 & $\pm 45,28$ & 31,86 & $\pm 38,90$ & 45,88 & $\pm 40,09$ & 35,22 & $\pm 41,52$ \\
\hline NP & 6,01 & $\pm 7,61$ & 19,9 & $\pm 29,41$ & 11,79 & $\pm 11,51$ & 8,64 & $\pm 11,57$ \\
\hline $\mathrm{P}$ & 0,13 & $\pm 0,34$ & 2,79 & $\pm 5,31$ & 2,34 & $\pm 3,42$ & 1,59 & $\pm 3,90$ \\
\hline MT & 6,14 & $\pm 7,74$ & 22,69 & $\pm 32,48$ & 14,12 & $\pm 13,99$ & 10,23 & $\pm 14,98$ \\
\hline Rapid & 0,06 & $\pm 0,17$ & 0,72 & $\pm 1,33$ & 0,94 & $\pm 1,28$ & 0,63 & $\pm 1,65$ \\
\hline Med & 0,1 & $\pm 0,23$ & 2,7 & $\pm 4,91$ & 1,59 & $\pm 2,48$ & 1,1 & $\pm 2,64$ \\
\hline Slow & 5,98 & $\pm 7,58$ & 19,27 & $\pm 28,32$ & 11,6 & $\pm 11,34$ & 8,51 & $\pm 11,26$ \\
\hline VCL & 11,23 & $\pm 13,16$ & 16,28 & $\pm 17,31$ & 18,82 & $\pm 18,30$ & 12,66 & $\pm 15,67$ \\
\hline VSL & 6,42 & $\pm 8,03$ & 7,38 & $\pm 9,78$ & 9,21 & $\pm 10,62$ & 6,18 & $\pm 8,52$ \\
\hline VAP & 7,93 & $\pm 9,53$ & 9,95 & $\pm 11,85$ & 11,56 & $\pm 12,93$ & 8,03 & $\pm 10,63$ \\
\hline LIN & 25,47 & $\pm 30,76$ & 23,06 & $\pm 23,69$ & 26,19 & $\pm 26,56$ & 21,28 & $\pm 25,06$ \\
\hline STR & 35,93 & $\pm 41,78$ & 38,76 & $\pm 37,60$ & 46,29 & $\pm 40,08$ & 34,3 & $\pm 39,56$ \\
\hline ALH & 0,44 & $\pm 0,93$ & 1,43 & $\pm 1,81$ & 1,47 & $\pm 1,85$ & 1,22 & $\pm 1,62$ \\
\hline BCF & 1,39 & $\pm 2,74$ & 4,11 & $\pm 4,94$ & 4,56 & $\pm 5,49$ & 4,14 & $\pm 5,03$ \\
\hline
\end{tabular}

T1: incidência de radiação vermelha antes do resfriamento e após a descongelação; T2: incidência de radiação vermelha apenas antes do resfriamento; T3: incidência de radiação apenas após a descongelação; GC: sem incidência de radiação vermelha. Static - espermatozoides estáticos; NP - motilidade não progressiva; $\mathrm{P}$ - motilidade progressiva; MT - motilidade total; Rapid - espermatozoides rápidos; Med - espermatozoides com velocidade média; Slow - espermatozoides com velocidade lenta; VCL - velocidade curvilinear; VSL - velocidade progressiva; VAP - velocidade média do trajeto; LIN - linearidade; STR - retilinearidade; ALH - amplitude do deslocamento lateral da cabeça; $\mathrm{BCF}$ - frequência de batimento flagelar. $(\mathrm{P}>0,05)$.

(Fonte: SCA® - Avaliação gerada pelos autores) 
Efeito da radiação...

Tabela 4. Médias ( \pm DP) dos parâmetros analisados pelo CASA, para espermatozoides caninos criopreservados, processados com e sem irradiação LED, 90 minutos após a descongelação

\begin{tabular}{crlrrlr}
\hline Características & \multicolumn{1}{c}{ T1 } & \multicolumn{1}{c}{ T2 } & \multicolumn{1}{c}{ T3 } & \multicolumn{1}{c}{ GC } \\
\hline Static & 24,97 & $\pm 42,77$ & 21,09 & $\pm 36,56$ & $37,4 \pm 43,20$ & $31,01 \pm 43,05$ \\
NP & 2,21 & $\pm 3,81$ & 5,75 & $\pm 11,03$ & $6,82 \pm 8,54$ & $5,1 \pm 7,17$ \\
P & 0,09 & $\pm 0,19$ & $0,43 \pm 1,17$ & $1,24 \pm 1,98$ & $0,25 \pm 0,43$ \\
MT & $2,3 \pm 3,97$ & $6,18 \pm 11,96$ & $8,06 \pm 10,31$ & $5,35 \pm 7,52$ \\
Rapid & 0,05 & $\pm 0,10$ & $0,11 \pm 0,35$ & $0,41 \pm 0,90$ & $0,1 \pm 0,18$ \\
Med & $0,07 \pm 0,18$ & $0,39 \pm 1,05$ & $0,99 \pm 1,48$ & $0,19 \pm 0,33$ \\
Slow & $2,18 \pm 3,75$ & $5,67 \pm 10,87$ & $6,65 \pm 8,31$ & $5,06 \pm 7,12$ \\
VCL & $6,07 \pm 10,64$ & $7,01 \pm 12,19$ & $14,54 \pm 17,51$ & $8,43 \pm 11,83$ \\
VSL & $2,33 \pm 4,01$ & $3,38 \pm 6,38$ & $7,08 \pm 8,85$ & $3,53 \pm 4,96$ \\
VAP & $3,22 \pm 5,58$ & $4,49 \pm 8,03$ & $9,24 \pm 11,29$ & $4,89 \pm 6,85$ \\
LIN & $10,81 \pm 18,96$ & $13,11 \pm 24,82$ & $21,72 \pm 25,37$ & $15,39 \pm 21,65$ \\
STR & $19,92 \pm 34,33$ & $19,7 \pm 34,41$ & $34,31 \pm 39,54$ & $26,29 \pm 36,64$ \\
ALH & $0,39 \pm 0,90$ & $0,61 \pm 1,28$ & $1,49 \pm 1,78$ & $0,89 \pm 1,56$ \\
BCF & $0,96 \pm 2,33$ & $1,8 \pm 3,50$ & $4,21 \pm 5,09$ & $2,86 \pm 4,93$ \\
\hline
\end{tabular}

T1: incidência de radiação vermelha antes do resfriamento e após a descongelação; T2: incidência de radiação vermelha apenas antes do resfriamento; T3: incidência de radiação apenas após a descongelação; GC: sem incidência de radiação vermelha. Static - espermatozoides estáticos; NP - motilidade não progressiva; P - motilidade progressiva; MT - motilidade total; Rapid - espermatozoides rápidos; Med - espermatozoides com velocidade média; Slow - espermatozoides com velocidade lenta; VCL - velocidade curvilinear; VSL - velocidade progressiva; VAP - velocidade média do trajeto; LIN - linearidade; STR - retilinearidade; ALH - amplitude do deslocamento lateral da cabeça; $\mathrm{BCF}$ - frequência de batimento flagelar. $(\mathrm{P}>0,05)$.

(Fonte: SCA® - Avaliação gerada pelos autores)

Aos 60 e 90 minutos após a descongelação, não foram observadas diferenças significativas entre os tratamentos, em nenhum dos parâmetros analisados pelo CASA (Tab. 3 e 4). Resultados similares foram encontrados por Brandão (2008), que, ao analisar os efeitos da radiação laser sobre o sêmen de equino fresco e descongelado, também não encontrou diferenças estatísticas entre os parâmetros avaliados pelo CASA nos espermatozoides irradiados antes ou após a congelação e naqueles sem irradiação. Porém, diferenças numéricas puderam ser observadas em alguns parâmetros analisados, os quais serão destacados a seguir.

Em relação à frequência de batimento flagelar (BCF), numericamente, pôde-se observar que aqueles espermatozoides que foram incididos somente após a descongelação mantiveram maiores valores desse parâmetro aos 60 e 90 minutos após a descongelação, sem, entretanto, ser significativa. Sabe-se que o BCF está intimamente relacionado às taxas de fertilização in vitro, sobretudo nos estágios iniciais da fecundação (Silva et al., 2006). Brandão (2008), no entanto, em seu estudo com sêmen equino, sugeriu que o espermatozoide equino tem melhor aproveitamento da energia produzida pela célula quando apenas se incide radiação vermelha antes do resfriamento.

Em todos os tratamentos, observou-se queda de todos os parâmetros analisados pelo CASA ao longo do tempo de TTR, como também foi observado por Laffaldano et al. (2005), quando do armazenamento de sêmen de peru, e por Brandão (2008), quando da avaliação do sêmen equino descongelado. Essa queda pode ser atribuída a uma baixa resistência térmica do espermatozoide de cão, já relatada por outros autores, que não está ligada diretamente a baixas taxas de fertilidade (Olar, 1984; Strom et al., 1997). Nesse período, também ocorre a redução ou esgotamento de ATP decorrente do metabolismo espermático. Porém, ao serem analisados numericamente os parâmetros de velocidade do espermatozoide (VCL, VSL e VAP), descritos por Verstegen et al. (2002) e por Silva et al. (2006) como significativamente relacionados ao número de espermatozoides capazes de penetrar a zona pelúcida do oócito, ou seja, indicativos da capacitação espermática, pôde-se observar uma queda mais lenta desses parâmetros naqueles espermatozoides irradiados 
apenas antes do resfriamento (Tratamento 3) (Fig. 1, 2 e 3). Esse dado corrobora os apresentados por Laffaldano et al. (2005), que afirmaram que aplicação do laser, numa dose de $3,24 \mathrm{~J} / \mathrm{cm}^{2}$ em sêmen de peru armazenado por 60

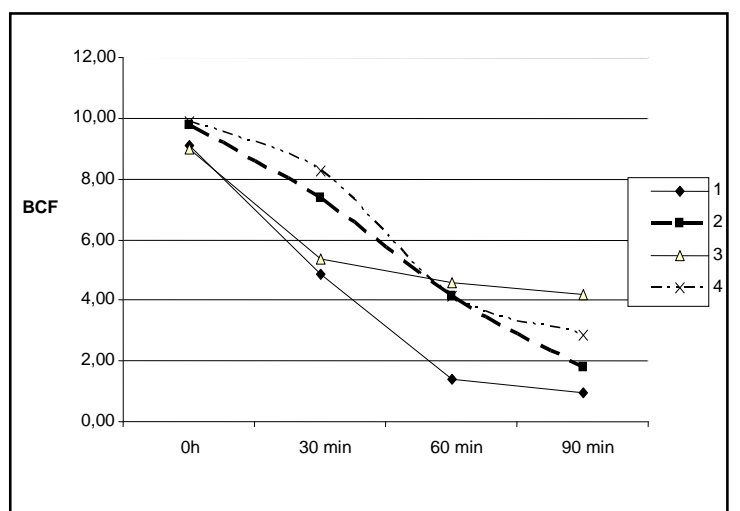

Figura 1. Valores da frequência de batimento flagelar (BCF) ao longo do TTR, analisado pelo CASA, nos diferentes tratamentos de incidência de radiação vermelha de baixa intensidade.

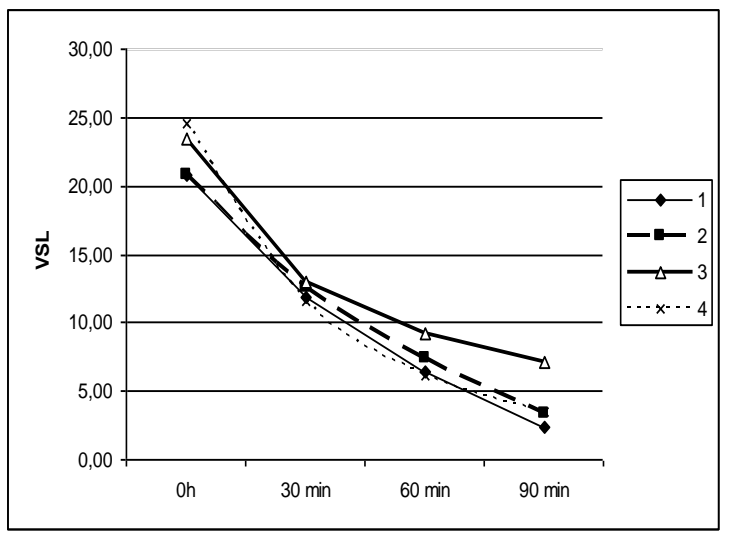

Figura 3. Valores da velocidade linear progressiva (VSL) ao longo do TTR, analisado pelo CASA, nos diferentes tratamentos de incidência de radiação vermelha de baixa intensidade. horas, reduz a deterioração dos espermatozoides ao longo do tempo, tendo, portanto, efeito positivo na longevidade dos espermatozoides dessa espécie, fato que deve ser mais estudado em se tratando do efeito da aplicação do LED.

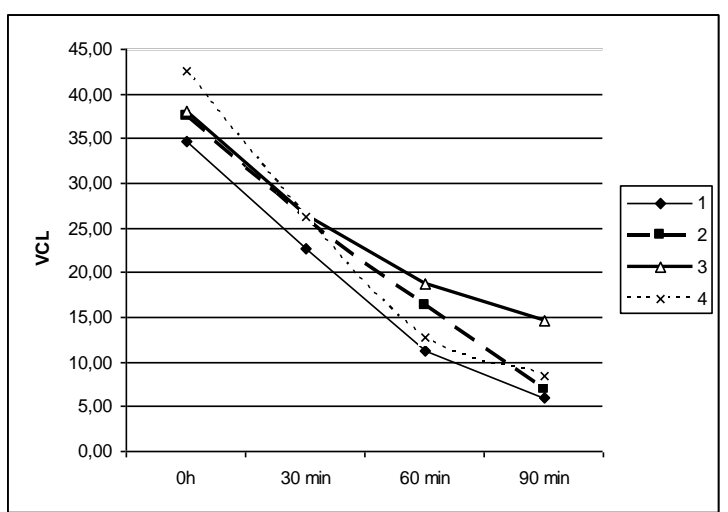

Figura 2. Valores da velocidade curvilinear (VCL) ao longo do TTR, analisado pelo CASA, nos diferentes tratamentos de incidência de radiação vermelha de baixa intensidade.

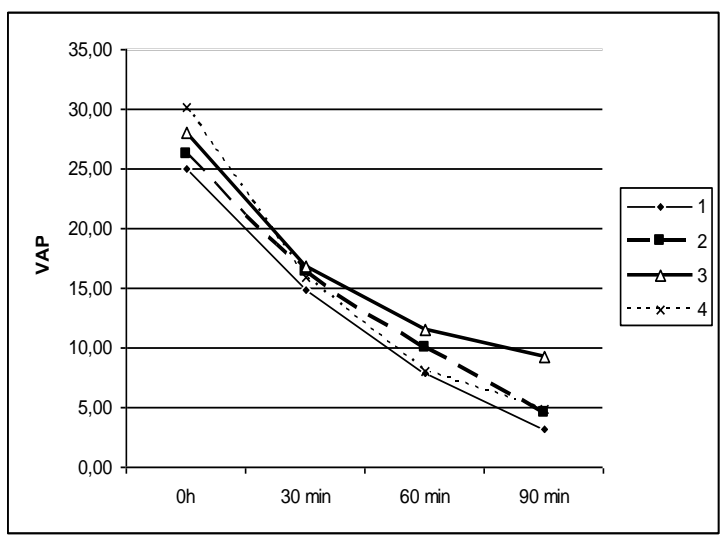

Figura 4. Valores da velocidade média do trajeto (VAP) ao longo do TTR, analisado pelo CASA, nos diferentes tratamentos de incidência de radiação.

BCF - batimento flagelar cruzado, VCL - velocidade curvelinear, VSL - velocidade linear progressiva, VAP - velocidade média do trajeto. (Fonte: Avaliação gerada pelos autores).

Brandão (2008) relata que a incidência de laser antes da congelação foi capaz de manter as características da célula durante o período de incubação, sugerindo que esse tratamento proporcionaria aos espermatozoides maior capacidade de suportar os estresses da criopreservação.
De forma similar, Corral-Baqués et al. (2005) demonstraram que o laser parece manter as características funcionais (VAP) do sêmen de cão, irradiado com diferentes doses de laser He$\mathrm{Ne}$, por pelo menos 45 minutos após a irradiação. 
Todas as discrepâncias observadas nos diferentes estudos mostram que ainda não há um conhecimento claro dos efeitos da radiação de baixa intensidade sobre a célula espermática, bem como do seu comportamento nas diferentes espécies animais. Portanto, são ainda necessários muitos estudos para esclarecer pontos importantes da aplicação da técnica. Da mesma forma são necessários outros estudos que empreguem o LED como fonte de radiação, uma vez que esse se apresenta como uma alternativa mais econômica e de menor consumo de energia em relação aos aparelhos de emissão LASER tradicionais.

\section{CONCLUSÕES}

A dupla incidência de radiação vermelha de baixa intensidade $(660 \mathrm{~nm})$, emitida por aparelho de LED de $100 \mathrm{~mW}$ de potência na dose de $6 \mathrm{~J} / \mathrm{cm} 2$ por 60 segundos em duas incidências seguidas, antes do resfriamento e após a descongelação, teve efeito deletério sobre a motilidade total do espermatozoide canino aos 30 minutos no teste de termorresistência, além de aumentar o número de espermatozoides estáticos, não progressivos e lentos; porém, não teve efeito significativo sobre as características cinéticas do sêmen de cães, analisadas pelo CASA, nos tempos de leitura imediatamente após a descongelação, 60 minutos e 90 minutos após a descongelação.

\section{AGRADECIMENTOS}

À Fundação de Amparo à Pesquisa, pela concessão de bolsa de auxílio, por meio do programa PROBIC/FAPEMIG PUC Minas.

\section{REFERÊNCIAS}

BRANDÃO, A.C. Efeito do laser diodo sobre as características de motilidade, de integridade de membranas plasmática e acrossomal e de pontencial de membrana mitocondrial de espermatozoides criopreservados de equinos. 2008. 87f. Tese (Doutorado em Medicina Veterinária) - Faculdade de Medicina Veterinária e Zootecnia, Universidade de São Paulo, São Pulo.
BUENO, R. Criopreservação de sêmen canino, utilizando dois diluidores e dois protocolos de resfriamento. 2000. 91f. Dissertação (Mestrado em Medicina Veterinária) - Escola de Veterinária, Universidade Federal de Viçosa, Viçosa - MG.

COHEN, N.; LUBART, R.; RUBINSTEIN, S.; BREITBART, H. Light irradiation of mouse spermatozoa: Stimulation of In Vitro Fertilization and Calcium Signals. Photoch. and Photob,. v.3, p.407-413, 1998.

CORRAL-BAQUÉS, M.I.; RIGAU, T.; RIVERA, M. et al. Effect of 655-nm diode laser on dog sperm motility. Las. in Med. Sci., v.20, p.28-34, 2005.

CORRAL-BAQUÉS, M.I.; RIVERA, M.M.; RIGAU, T. et al. The effect of low-level laser irradiation on dog spermatozoa motility is dependent on laser output Power. Las. in Med. Sci., v.24, p.703-713, 2009.

LAFFALDANO N.; MELUZZI, A.; MANCHISI, A.; PASSARELLA, S. Improvement of stored turkey semen quality as a result of $\mathrm{He}-\mathrm{Ne}$ laser irradiation. Anim. Reprod. Sci., v.85, p.317-325, 2005.

LAFFALDANO, N.; ROSATO, M.P.; PAVENTI, G. et al. Efecto de la radiación con laser de $\mathrm{He}-\mathrm{Ne}$ sobre la motilidad y los parámetros bioquímicos de espermatozoides de conejo durante su conservación. In: CONGRESSO IBERICO DE CUNICULTURA, 2., 2007, Vila Real Portugal. p.33-36.

MANUAL para exame andrológico e avaliação de sêmen animal. 2.ed. Belo Horizonte: Colégio Brasileiro de Reprodução Animal, 1998. 49p.

OCAÑA-QUERO, J.M.; GOMEZ-VILLAMANDOS, R.; MORENO-MILLAN, M.; SANTISTEBANVALENZUELA, J.M. Biological effects of heliumneon (He-Ne) laser irradiation on acrosome reaction in bull sperm cells. J. of Photoch. and Photob. B: Biology, v.40, p.294-298, 1997.

OLAR, T.T.; BOWEN, R.A.; PICKETT, B.W. Influence of extender, cryopreservative and seminal processing procedures on post-thaw motility of canine spermatozoa frozen in straws. Theriogenology, v.31, p.451-461, 1989. 
SILVA, A.R.; CASSIA, R.; CARDOSO, S. et al. Prognostic value of canine frozen-thawed semen parameters on in vitro sperm-oocyte interactions. Theriogenology, v.66, p.456-462, 2006.

SILVA, A.R.; CARDOSO, R.C.S.; UCHOA, D.C.; SILVA, L.D.M. Quality of canine semen submitted to single or fractionated glycerol addition during the freezing process. Theriogenology, v.59, p.821-829, 2003.

STROM, B.; ROTA, A.; LINDE-FORSBERG, C. In vitro characteristics of canine spermatozoa subjected to two methods of cryopreservation. Theriogenology, v.48, p.247-256, 1997.
VALLE，G.R.; ANDRADE，C.R.G.; SILVA, L.C.; VIANNA, L.R. Utilização de cones de polietileno para coleta de sêmen canino. Rev.Bras. Reprod. Anim., v.36, p.250-255, 2012.

VERSTERGEN, J.; IGUER-OUADA, M.; ONCLIN, K. Computer assisted sêmen analyzers in andrology research and veterinary practice. Theriogenology, v.57, p.149-179, 2002. 